\title{
Some effects of epistemological structure on memory*
}

\author{
JAMES D. HOLLAN $\dagger$ \\ Clarkson College of Technology, Potsdam, New York 13676
}

\begin{abstract}
Graph theoretical models of the epistemological structure imposed upon a set of mathematical concepts by Ss' definitions of them were constructed. Three graphical characteristics of the models were manipulated in a series of experiments to assess their effects on recall. Experiment I showed that recall and confidence of recall were highest for concept pairs connected by short paths, next highest for those connected by long paths, and lowest for those not connected by paths. Experiment II replicated the result of Experiment I that recall and confidence of recall were greater for concept pairs connected by paths than for those not connected by paths and revealed no differences in recall between concept pairs connected by many paths and those connected by a few paths. Experiment III found no differences in recall of abstract and concrete concepts. The abstractness of a concept was quantified by the mean path length from the concept to primitive (undefined) concepts. The approach of building a model of each S's epistemological structure was supported by the finding that, while a given S's definitions of the concepts remained relatively constant, there were considerable differences in definitions of the concepts between Ss.
\end{abstract}

Currently there is a burgeoning interest in semantic relationships and their importance to memory. Much of this interest can be traced to the work of Quillian (1966) on semantic memory, and most experimental studies in this area have followed the paradigm set forth by Collins and Quillian (1969).

This paper is concerned with the semantic relation of definition and with the effects on memory of various aspects of the epistemological structure imposed upon a set of concepts by this relation. The present experiments contrast with previous work in semantic memory by computing, rather than assuming, the particular epistemological structuring of the set of stimulus concepts for each $S$ and by employing recall rather than reaction time tasks.

\section{THE EPISTEMOLOGICAL STRUCTURE}

A concept's definition states the essential characteristics of the concept's referent and thus serves epistemologically to distinguish the referent from every thing else. A concept is logically dependent upon the concepts which comprise its definition, and these concepts are, in turn, dependent upon the concepts which comprise their definitions, etc. Thus, inherent in any set of concepts is a dependency structure imposed upon the concepts by their definitions.

This epistemological structure can be graphically represented by a digraph in which a point of the graph is associated with each concept and a directed edge from one point to another point exists just in case the concept

\footnotetext{
* This paper is based on a dissertation submitted to the Department of Psychology, University of Florida, in partial fulfilment of the requirements for the PhD degree. The author expresses his appreciation to his dissertation committee and to R. E. Osteen for allowing his computer program, PROJECT_X to be adapted to the requirements of this study.

tRequests for reprints should be sent to the author at the Department of Social Sciences, Clarkson College of Technology, Potsdam, New York 13676.
}

associated with the first point is defined in terms of the concept associated with the second point. Because of the complexity that can arise in the epistemological structure associated with even a few concepts, a computer program was used to construct the epistemological structures of the $S$ s in the present experiments and to ascertain the many characteristics of these structures made possible by the graphical formalization.

The input to the program employed is a set of concepts, the definitions of the concepts, and key concepts from the definitions. The program forms the epistemological structure imposed upon the set of concepts by the relation of definition and outputs the following information: all the paths (Harary, 1969) from each concept to primitive (undefined) concepts, an alphabetical list of the concepts which includes each concept's fathers and sons (i.e., the concepts which this concept is defined in terms of and the concepts which are defined in terms of this concept), and all paths that exist between every pair of concepts with their length.

\section{OVERVIEW OF THE EXPERIMENTS}

By formalizing, in graph theoretic terms, the epistemological structure imposed upon a set of concepts by their definitions, it becomes possible to ascertain many structural characteristics of this epistemological structure. Once these characteristics have been identified, it is then possible to experimentally investigate their effect upon performance in psychological tasks in which such epistemological information is thought to be important.

The general strategy of the experiments reported here is to identify the structural characteristics of a S's epistemological structuring of a set of concepts and then to vary the concepts presented in memory tasks in such a way that the effect upon memory recall of these 
structural characteristics can be assessed.

I chose to use mathematicians as Ss in this study. This choice was dictated primarily by the fact that it allowed the use of mathematical concepts as the stimulus materials in the experiments The motivation to use mathematical concepts was that these concepts are, in general, much more precisely formulated than concepts from other areas and are, thus, ideally suited to the construction of their associated epistemological structure.

The choice of mathematicians as Ss also allowed the restriction of the set of concepts used in the experiments to a rather limited set, namely, those of naive set theory (Halmos, 1960), with the assurance that since most mathematicians are well acquainted with set theory, the familiarity of the Ss with the set of concepts used was reasonably uniform.

Fifty concepts were chosen from a text on set theory (Halmos, 1960), and each S was asked to define these concepts. The epistemological structure imposed on the set of concepts by a S's definitions of them was constructed by use of the computer program mentioned earlier. Thus, each S's particular epistemological structuring of the set of concepts was known along with the structural characteristics associated with each concept as determined by the graphical formulation of the structure.

In each of three experiments, one characteristic of a S's epistemological structure was manipulated, by appropriate selection of the concepts presented to the $S$, and its effect upon memory recall was assessed. The particular characteristics studied were the number of paths between pairs of concepts, the lengths of such paths, and the abstractness of the concepts. For purposes of this study, the abstractness of a concept was defined to be the mean path length of the concept from the primitive concepts. A paired-associate paradigm was employed to investigate the number and length of paths between concept pairs, while a free recall paradigm was used in the investigation of the abstractness of the concepts.

\section{EXPERIMENT I}

\section{Method}

Subjects. Ten graduate students and faculty members of the University of Florida Department of Mathematics participated in the experiment. Each S was paid for his participation in this and the following experiments.

Design. For each S, 21 pairs of concepts were formed based upon his constructed epistemological structure. A given pair of concepts was connected in the graphical representation of a S's epistemological structure by a short path (mean length of 1.06 and standard deviation of 0.23 ), a long path (mean length of 3.86 and standard deviation of 0.69 ), or was not connected by any path. A within-S design was employed crossing distance between concepts with trials.

Procedure. For each S, five random mixed-list orders of the S's 21 pairs of concepts ( 7 of each type) were constructed with the restriction that a proportionate number of the three types of pairings occurred in each quarter of an ordering. The 21 pairs of concepts were presented tachistoscopically to a $S$ at the rate of 1 pair every $8 \mathrm{sec}$ with a pair visible for $2 \mathrm{sec}$. After exposure to the 21 pairs of concepts, a $S$ participated in a decoding task for 2 min to preclude recall from short-term memory. A S was then given a sheet of paper containing all of the left-hand members of the pairs of concepts and asked to write next to each concept the associated right-hand member and to give an assessment of his confidence of correct recall on a 4-point scale. Four such study-test trials were completed in an experimental session. After a period of $24 \mathrm{~h}$, each $\mathrm{S}$ retumed and was given a test trial followed by a study-test trial.

\section{Results}

Analysis of Mean Percentage Correct Recall. The mean percentage correct recall of the response terms (MPCRT) for each distance condition was computed for each $S$ on each trial. A two-factor repeated measures analysis of variance performed on this measure revealed that the overall effects of distance, of trials, and of the interaction between distance and trials were significant $(p<.001)$. Figure 1 depicts the MPCRT on the six trials for each distance condition.

Because of the significant interaction between distance and trials and because the distance variable was of primary interest in this experiment, Tukey's HSD test was used to compare the differences between the means of each level of the distance variable on each trial. This analysis revealed that on the first two trials, the MPCRT of concept pairs connected by short, long, or no paths were significantly $(\mathrm{p}<.01)$ different from each other with recall highest for short pairs, next highest for long pairs, and lowest for unconnected pairs; that MPCRT of short pairings were significantly $(p<.05)$ larger than those for long pairings on Trials 3 and 5; and that the MPCRT for short pairs were significantly $(p<.01)$ greater than for pairs not connected by a path on Trial 3.

Analysis of Mean Confidence Rating. For each trial, each S's mean confidence rating for recall of the response terms in each distance condition was computed. As with the analysis of MPCRT, the effects of distance, of trials, and of their interaction were all significant $(p<.001)$. Figure 2 shows the mean confidence rating on the six trials for each type of concept pairing.

Tukey's HSD test was used to make all pairwise comparisons between the means of each level of the distance variable on each trial. The results of the HSD test almost exactly parallel those of the MPCRT analysis. On Trials 1 and 2, Ss were significantly ( $p<.01$ and $p<.05$, respectively) more confident of their recall of response terms of concept pairs connected by short paths than those not connected by any paths or those connected by long paths, and the confidence of recall of response terms of long pairings was significantly $(p<.01)$ higher than that of those not connected by paths. Other significant $(p<.01)$ differences were found on Trials 3 and 5 with confidence of recall for the short pairings greater than that for long pairings on Trial 5 and for those pairings not connected by any paths on Trial 3 . 


\section{Discussion}

The effect upon recall of three different levels of semantic relatedness of concept pairs was experimentally investigated, using a new approach which was designed to make possible greater control of this variable. In a paired-associate task, pairs of concepts which were connected by long paths, short paths, or were not connected by any paths were employed. Semantic distance, as quantified by mean path length between pairs of concepts in the graphical representation of the epistemological structure that a $S$ imposed on a set of concepts by his definition of them, was found to be a potent variable affecting memory.

The results were in accordance with a straightforward extrapolation from reaction time studies of semantic memory: recall and confidence of recall were highest for response terms of concept pairs connected by short paths, next highest for those of concept pairs connected by long paths, and lowest for response terms of concept pairs not connected by any paths. Although this ordering of recall was found only on the initial trials, parallel results were obtained from the analysis of the two dependent variables: mean percentage correct recall and mean confidence rating.

While there were no significant differences between either recall or confidence of recall for response terms of concept pairs not connected by paths and those of concept pairs connected by long paths subsequent to the second trial, it is interesting to note that as late as the fifth trial, i.e., the first delayed trial, there was a significant difference for recall and confidence of recall

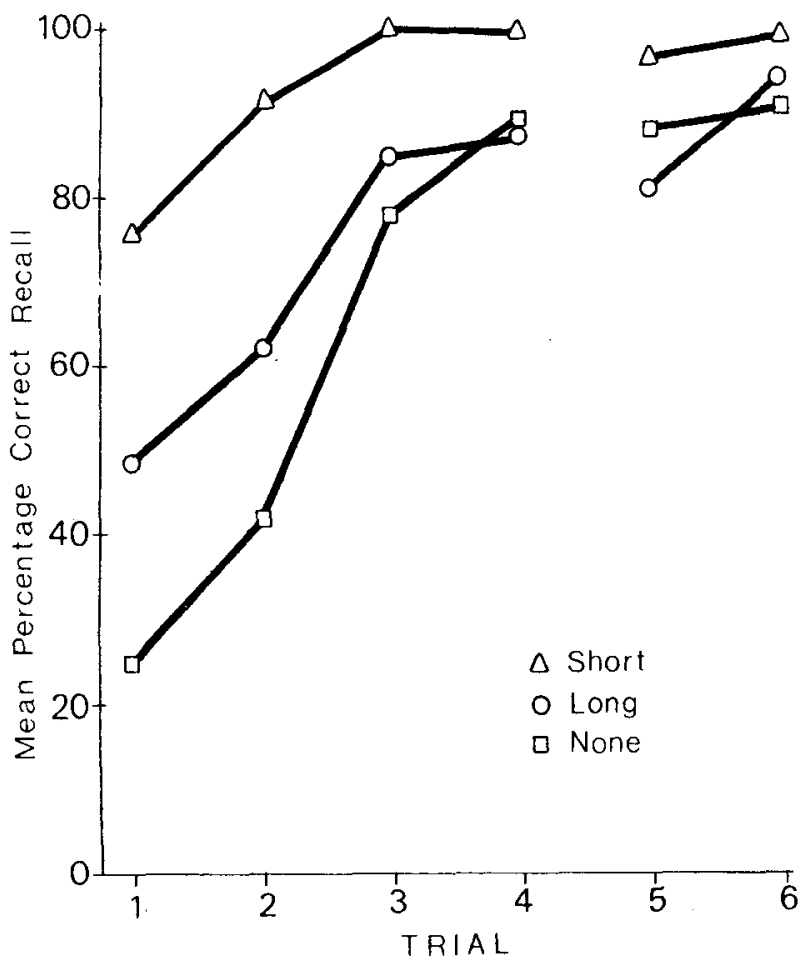

Fig. 1. Mean percentage correct recall on each trial for the three distance conditions.

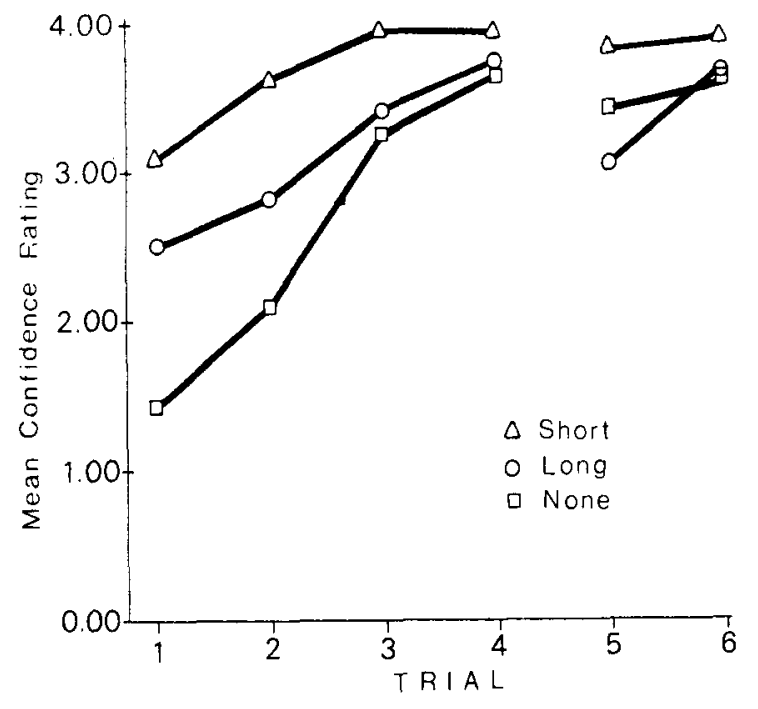

Fig. 2. Mean confidence rating on each trial for the three distance conditions.

for response terms of concept pairs connected by short paths and those of concept pairs connected by long paths. One can reasonably postulate, if retrieval strategies make use of the structural aspects of the epistemological structure, that Ss adopt different strategies for recall of response terms of concept pairs connected by paths and those not connected by paths. Based on this assumption, the results indicate that the strategy for pairs connected by paths was, in accordance with reaction time studies, more effective, even as late as the fifth trial, for those pairs connected by short paths, while the strategy for unconnected pairs was, subsequent to the third trial, an equally effective recall strategy. It can, on the other hand, be argued that the failure of a continuation of the clear distinction between recall for the three different types of pairings, following the second trial, was due to nothing more than a ceiling effect of approaching perfect recall. In any case, the results of the analyses of recall and confidence of recall for response terms of concept pairs connected by paths are in agreement with Quillian's (1969) model of semantic memory and contribute evidence for semantic distance being a variable of importance in memory tasks involving concepts.

In regard to any conclusions concerning the relative effectiveness of the retrieval strategy used with concepts connected by paths and the strategy used with concepts not connected by paths, if one accepts the assumption of different strategies, one point should be made clear. The former strategy, because of its dependence on the meanings, i.e., the definitions, of the concepts, is one that would readily be adopted by a $S$, since it is the meaning of concepts which the $S$ normally deals with. If this is, in fact, the case, the inferiority of recall on the initial trials for response terms of concept pairs not connected by paths could be accounted for by the S's initially attempting to adopt this type of strategy and, 


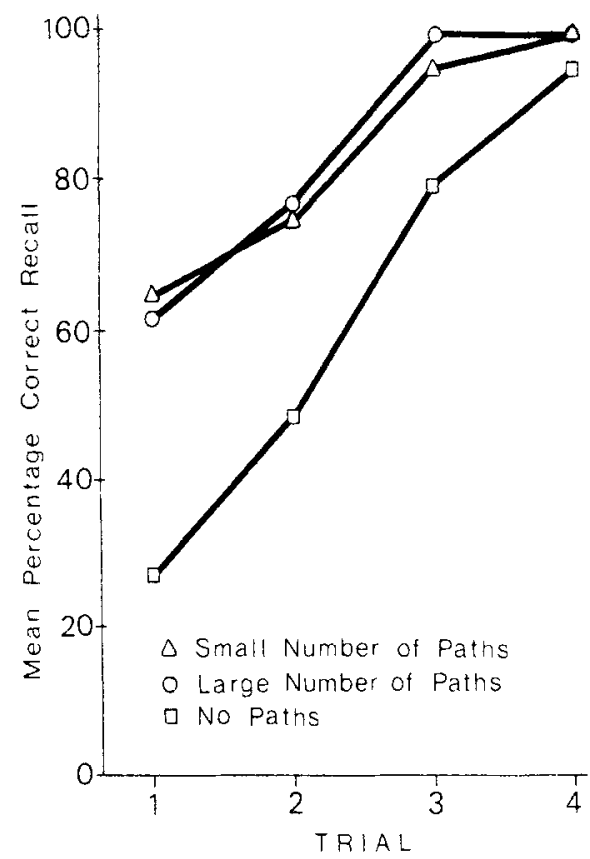

Fig. 3. Mean percentage correct recall on each trial for the three levels of the path condition.

because of the lack of such relationships between the concepts, as evidenced by the graphical representation, finding it unsuccessful, turning to some other recall strategy based on other aspects of the concepts.

Whether or not different recall strategies were employed by Ss in dealing with the different types of pairs, it seems clear that the graphical representation of the epistemological structure a $\mathrm{S}$ imposes on a set of concepts by his definitions of them has led to the quantitative identification of a relationship between concepts, semantic distance, which is an important variable in the type of memory tasks used in this experiment.

\section{EXPERIMENT II}

\section{Method}

Subjects. The same 10 graduate students and faculty members of the Department of Mathematics served as $\mathrm{Ss}$ in this experiment.

Design. For each S, 21 pairs of concepts were formed based on his constructed epistemological structure. A given pair of concepts was connected in the graphical representation of a S's epistemological structure by a small number of paths (mean of 1.12 and standard deviation of 0.33 ), by a large number of paths (mean 6.97 and standard deviation of 3.95 ), or by no paths. The set of concepts did not intersect with the set used in Experiment I, and an attempt was made to hold the distance between concepts connected by paths constant. A within-S design was employed crossing the two factors: number of paths between concepts and trials.

Procedure. For each S, four random mixed-list orders of the S's 21 pairs of concepts ( 7 pairs from each condition) were constructed with the restriction that a proportionate number of the three types occurred in each quarter of an ordering. The 21 pairs of concepts were presented tachistoscopically to a $S$ at the rate of 1 pair every $8 \mathrm{sec}$ with a pair visible for $2 \mathrm{sec}$. After exposure to the 21 pairs of concepts, a $S$ participated in a decoding task for $2 \mathrm{~min}$ to preclude recall from short-term memory. A $S$ was then given a sheet of paper containing all of the left-hand members of the pairs of concepts and asked to write next to each concept the associated right-hand member of the pair and to give an assessment of his confidence on a 4-point scale. Four such study-test trials were completed in the experimental session.

\section{Results}

Analysis of Mean Percentage Correct Recall. The MPCRT for each level of the number of paths variable was computed for each $S$ on each trial. A two-factor repeated measures analysis of variance revealed a significant $(p<.01)$ overall effect of number of paths, trials, and a significant $(\mathrm{p}<.05)$ interaction between trials and number of paths. Figure 3 depicts the MPCRT for the four trials for each type of concept pairing.

As in the first experiment, Tukey's HSD test was employed to make all pairwise comparisons between the MPCRT for each number of paths on each trial. It was found that there was no significant $(p>.05)$ difference, either overall or on any trial, between MPCRT for concept pairs connected by a small number of paths and those connected by a large number of paths. It was also found that, both overall and on the first three trials, the MPCRT for pairs of concepts not connected by paths was significantly $(p<.05)$ less than those connected by paths.

Analysis of Mean Confidence Rating. For each trial, a S's mean confidence rating for recall of the response terms of the three types of concept pairings was computed. Figure 4 shows the mean confidence ratings on the four trials for each type of concept pairing. The results of an analysis of variance performed on these mean confidence ratings, as in the first experiment, paralleled those of the MPCRT analysis. The effects of

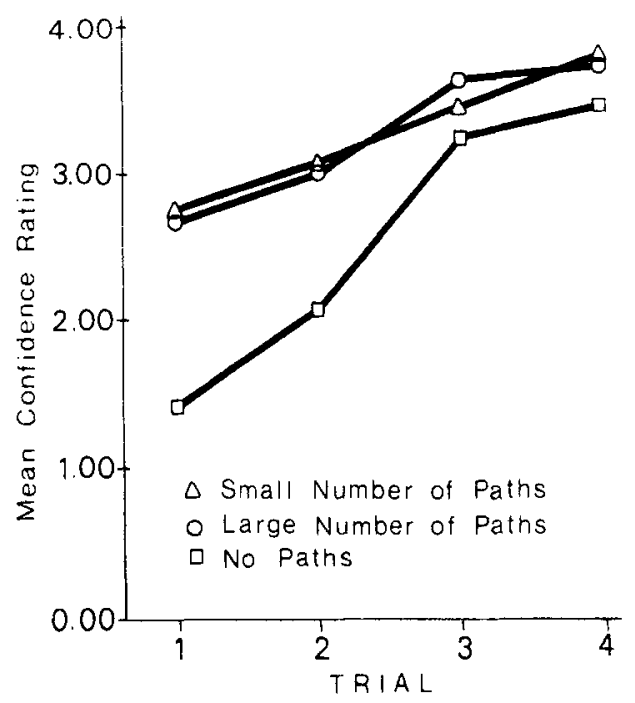

Fig. 4. Mean confidence rating on each trial for the three levels of the path condition. 
the number of paths, trials, and the interaction between number of paths and trials were significant $(p<.01)$.

Tukey's HSD test revealed that there was not a significant $(p<.05)$ difference, either overall or on any trial, between the mean confidence rating for recall of the response terms of concepts connected by a small number of paths and those of concepts connected by a large number of paths. As with the MPCRT analysis, the mean confidence ratings of Ss were significantly $(p<.05)$ higher for recall of response terms of concept pairs connected by either a small or large number of paths than for those not connected by any paths, both overall and on the first two trials.

\section{Discussion}

In this experiment, no differences were found for recall or confidence of recall of response terms of concept pairs connected by many paths and those connected by few paths. As in the first experiment, though, there was, on the initial trials, a significant superiority of recall and confidence of recall for response terms of concept pairs which were connected by paths over that of pairs not connected by paths. The results of the analyses of the two dependent variables again were parallel.

The strength of associative relationships between word pairs has been shown (see, for example, Jenkins, Mink, \& Russel, 1958) to be an important variable affecting recall in paired-associate tasks. One measure of strength of association devised by Deese (1962) is simply to count the number of associates a pair of words have in common.

There are some grounds for postulating that concept pairs with many paths between them would have more common associates than those with only a few connecting paths. This assumption would lead one to expect that recall of response terms of pairs connected by many paths would be superior to that of pairs connected by a few paths. The results of this experiment are not in accordance with this prediction.

It should be pointed out that the results of this experiment are in agreement with one aspect of Quillian's theory of semantic memory. Quillian (1969) contends that search procedures dealing with semantic memory proceed in parallel fanning out from a given concept in a "breadth-first" fashion. In terms of the graphical representation presented in this study, this type of search procedure would proceed along each path joining a pair of concepts in parallel. Therefore, the number of paths between a pair of concepts would not be a factor influencing recall.

\section{EXPERIMENT III}

\section{Method}

Subjects. The same 10 graduate students and faculty members of the Department of Mathematics served as Ss.

Design. For each $\mathrm{S}$, based on his epistemological structuring of the set of concepts, two nonintersecting sets of 10 concepts each

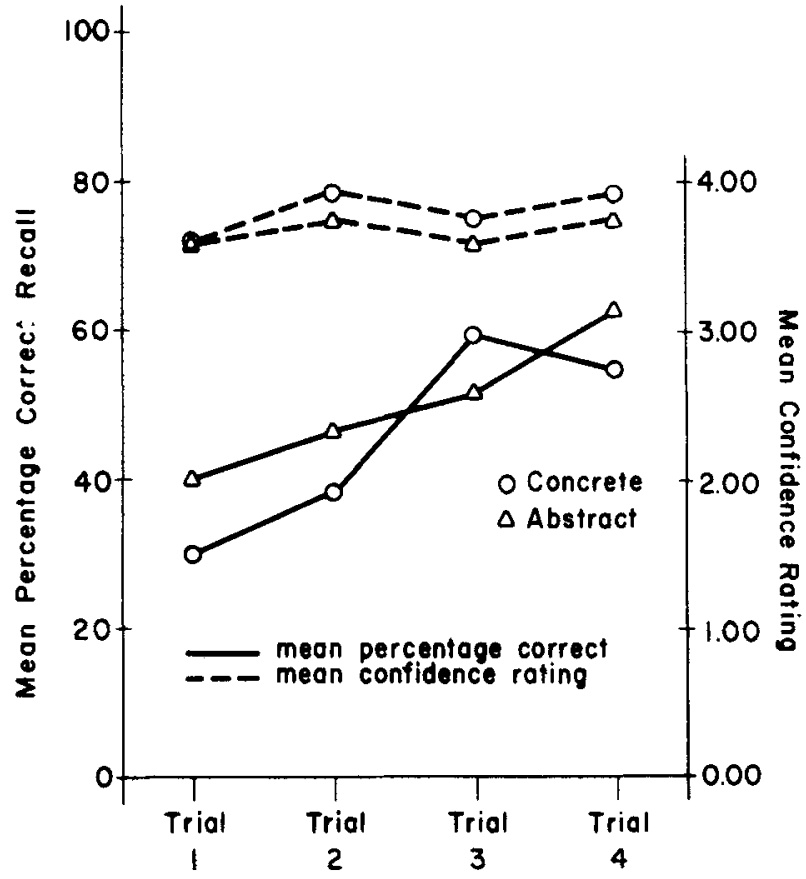

Fig. 5. Mean percentage correct recall and mean confidence rating on each trial for abstract and concrete concepts.

were identified such that the difference in mean path length from primitive concepts was maximized between the two sets. Across Ss, the mean of the mean path lengths from primitive concepts for one set was 0.95 with standard deviation of 0.36 and for the other the mean was 3.78 with standard deviation of 0.43. The former set of concepts will be termed "concrete," while the latter will be called "abstract." Examples, from one S's protocol, of concrete concepts are: singleton, ordered pair, and intersection. Examples, from the same S's protocol, of abstract concepts are: commutative, successor, and equivalence class. A free recall experiment employing a within-S design was used to ascertain the effects of abstractness and concreteness across four trials.

Procedure. For each S, four random lists of each S's 20 concepts were constructed with the restriction that a proportionate number of concrete and abstract concepts occurred in each quarter of a list.

On each of four trials, a $S$ was allowed to view one of his four lists for a period of $11 \mathrm{sec}$. Following each trial, a $S$ participated in a decoding task for $2 \mathrm{~min}$ to preclude recall from short-term memory. After the decoding task on each trial, a $\mathbf{S}$ was given a sheet of paper and asked to write all of the concepts which had been presented and next to each give an assessment of his confidence of correct recall on a 4-point scale.

\section{Results}

For each $\mathrm{S}$, the mean percentage of correct recall and mean confidence ratings for recall of abstract and concrete concepts on each trial were computed. These means are depicted in Fig. 5. A two-factor repeated measures analysis of variance, crossing the abstractness of the concepts with trials, was used to analyze the data.

The analyses revealed no significant $(F<1)$ differences, for either accuracy or confidence of recall, between abstract and concrete concepts. There was no significant interaction $(\mathrm{F}<1)$ between the abstractness of the concepts and trials, but the usual significant $(p<.01)$ trials effect was found. 
In order to quantify the extent of clustering of concrete and abstract concepts in the S's recall, the ARC index of clustering (Roenker, Thompson, \& Brown, 1971) was computed from the recall of each $S$ on each trial. The means of the ARC scores on Trials 14 were $.372, .131, .123$, and .197 with standard deviations of $.683, .515, .327$, and .270 , respectively. A single-factor repeated measures analysis of variance revealed no significant $(F<1)$ clustering differences between trials.

\section{Discussion}

In this experiment, abstract and concrete concepts were presented in randomized lists using a free recall paradigm. No differences were found for either recall or confidence of recall between the two types of concepts.

The abstractness of a given concept was quantified by the mean path length between the concept and the primitive concepts to which it was connected in the graphical representation of a S's epistemological structuring of the set of concepts. Thus, this quantification of the abstractness of a concept is dependent upon the definition of the concept.

One can speculate that the recall strategies employed by the Ss attempting to recall the lists of words primarily made use of nondefining characteristics of the concepts. Some verbal reports of the Ss were in accordance with this speculation. If this were the case, one would not expect to observe any difference between recall of abstract and concrete concepts. Following from this speculation, a more fruitful paradigm for assessing possible differential effects of the abstractness variable would be one which focused the Ss' recall strategies on the meanings of the concepts.

The ARC clustering scores were also in accordance with the above speculation about recall strategy. If the strategies employed by the Ss were dependent upon the factors which distinguished the two types of concepts, one would expect an increase of clustering across trials. This was not the case.

\section{CONSISTENCY OF EPISTEMOLOGICAL STRUCTURES}

Each S defined the set of 50 set-theoretic concepts on two occasions. The first occasion was before the start of the sequence of experiments and the second occasion was about 2 months later following the conclusion of the final experiment. In an effort to indicate the consistency of the Ss' epistemological structurings of the set of concepts, the percentage of agreement in defining the set of concepts was computed. The mean percentage of agreement within Ss in defining the set of concepts on the two occasions was 89.68 with standard deviation of 4.23. The mean percentage between Ss was 62.07 with standard deviation of 3.10 for the first occasion and 60.37 with standard deviation of 4.26 for the second occasion. Note therefore that while a given S's definitions of the set of concepts remained relatively constant, there were considerable differences in definitions between Ss.

\section{GENERAL DISCUSSION}

These investigations have been based on a rather limited graph theoretical model of epistemological structure: that imposed upon a set of concepts by their definitions. A computer program made possible the construction of each S's epistemological structuring of 50 concepts from set theory and the identification of many associated structural characteristics. A number of these graphical characteristics-the distance between pairs of concepts, the number of paths between concept pairs, and the abstractness of the concepts-were manipulated in order to assess the effects of these characteristics on recall.

The reasoning behind the experiments was the idea that if the model accurately represents some aspects of the epistemological information that a $S$ might use in memory tasks, then the quantitative identifications made possible by the model would allow one to manipulate variables which affect a S's performance in such memory tasks. The suitability of the model is rather straightforward when variations in graphical characteristics, by appropriate selection of stimulus materials, result in concomitant variations in performance, but rather difficult to evaluate when there are no variations in performance. In this latter case, it is a moot point whether such characteristics are invalid identifications or, while valid identifications, are not of differential importance to performance.

The major result of the studies reported here was that semantic distance as quantified by mean path length between concepts in the graphical representation of a S's epistemological structure was a potent variable affecting recall of concepts in a paired-associate task. A corollary of this result was that recall for response terms of concept pairs connected by paths was decisively better than those of concept pairs not connected by paths. Both of these results lend weight to the accuracy of the model of epistemological structure presented here; but it is necessary to mention that the present results could be accommodated by a number of current models of semantic structure (e.g., Quillian, 1969; Rumelhart, Lindsay, \& Norman, 1972; Anderson \& Bower, 1973; Collins \& Loftus, 1973).

As mentioned earlier, the negative findings are much more difficult to interpret. For example, it is usually possible to postulate a recall strategy based on the model which could account for any particular negative findings. Such postulations and speculations as offered here are, of course, only conjectures and should be subjected to further experimental analysis.

The positive findings of these studies not only provide evidence in support of the particular model of epistemological structure presented here, but also for the distinctive approach of these investigations. This 
approach was based on the assumption that each S's particular structuring of the set of concepts was of primary importance to his subsequent performance in memory tasks involving these concepts. Note as supportive evidence for this approach the high level of agreement of definitions within Ss but the comparatively low level of agreement between Ss. This approach contrasts greatly with that of other researchers in the area of semantic memory. A recent study (Rips, Shoben, \& Smith, 1973) has departed somewhat from the typical approach of assuming some normative structure (e.g., dictionary definitions) to be an adequate model of each S's epistemological structure. These investigators measured semantic distance by asking Ss to indicate the degree of relatedness, on a 4-point scale, of pairs of words. They found that rated distance and a derived distance, based upon a multidimensional scaling technique, were predictive, under certain conditions, of reaction times in verification of semantic relationships involving these words. In the Rips et al (1973) study, there is an attempt to directly measure semantic distance, and this provides greater control of this variable. The approach of the present experiments was chosen because of the considerable experimental control it afforded. It may be the case that lack of such control might well have led to the present controversies (e.g., Wilkins, 1971; Conrad, 1972) surrounding the reaction time studies discussed earlier. In any case, when dealing with a phenomenon as complex and intractable as human memory, any additional control over the experimental situation is of tremendous importance.

The major benefit of this model of epistemological structure was that it allowed the quantification of numerous structural characteristics by virtue of its graphical formulation. Such quantifications made possible the experimental investigations which comprise this work. There are numerous other things which a model of epistemological structure makes possible. It allows one to formulate in explicit terms recall strategies which a $\mathrm{S}$ might use in retrieving information from semantic memory, e.g., Quillian's (1969) breadth-first search. It allows the identification of the many logical dependencies which are contained in the epistemological structure. These dependencies, while important in their own right, might well be important to cognitive activities like memory, but such importance is unlikely to be identified without models of this structure and computerized techniques for accessing structural characteristics. In addition, a graphical model allows the use of the extensive literature of graph theory in identifying the structural characteristics. Finally, and possibly most importantly, to the extent that the model is an accurate representation of a S's epistemological structure, it allows an extensive analysis of the structural characteristics of this structure, without requiring the continual presence of the $S$, freeing the investigator of many restrictions usually associated with human Ss, and thus might well make possible much better controlled and informative subsequent experiments.

\section{REFERENCES}

Anderson, J. R., \& Bower, G. H. Human associative memory. Washington, D.C: Winston, 1973.

Collins, A. M, \& Loftus, E. F. A spreading activation theory of semantic processing. Report No. 2711, Bolt Beranek and Newman, Inc., 1973.

Collins, A. M., \& Quillian, M. R. Retrieval time from semantic memory. Journal of Verbal Learning \& 'Verbal Behavior, $1969,8,241-248$.

Conrad, C. Cognitive economy in semantic memory. Journal of Experimental Psychology, 1972, 92, 149-154.

Deese, J. On the structure of associative memory. Psychological Bulletin, 1962, 69, 161-175.

Halmos, P. R. Naive set theory. Princeton, N.J: Van Nostrand, 1960.

Harary, F, Graph theory. Reading, Mass: Addison-Wesley, 1969. Jenkins, J. J., Mink, W. D., \& Russel, W. A. Associa tive clustering as a function of verbal associative strength. Psychological Reports, 1958, 4, 127-136.

Quillian, M. R. Semantic memory. PhD dissertation, Carnegie Institute of Technology, 1966.

Quillian, M. R. The teachable language comprehender. Communications of the ACM, $1969,12,459-476$.

Rips, L. J., Shoben, E. J., \& Smith. E. E. Semantic distance and the verification of semantic relations. Journal of Verbal Learning \& Verbal Behavior, 1973, 12, 1-20.

Roenker, D. L., Thompson, C. P., \& Brown, S. C. A comparison of measures for the estimation of clustering in free recall. Psychological Bulletin, 1971, 76, 45-48.

Rumelhart, D. E., Lindsay, P. H., \& Norman, D. A. A process model for long-term memory. In E. Tulving and W. Donaldson (Eds.), Organization and memory. New York: Academic Press, 1972 .

Wilkins, A. T. Conjoint frequency, 'category size, and categorization time. Journal of Verbal Learning \& Verbal Behavior, $1971,10,382 \cdot 385$.

(Received for publication January 19, 1974; revision received April 2, 1974.) 